\title{
Reflectance Confocal Microscopy Applied to Folliculitis Decalvans: Preliminary Results of a Multicenter Study
}

\author{
Sara Mazzillia Laura Vollono ${ }^{a}$ Francesco Tassone $^{\mathrm{b}, \mathrm{c}}$ Simone Cappilli ${ }^{\mathrm{b}, \mathrm{c}}$ \\ Ketty Peris ${ }^{b, c}$ Luca Bianchi ${ }^{a}$ \\ aDermatology Unit, University of Tor Vergata, Rome, Italy; 'bnstitute of Dermatology, Università Cattolica, \\ Rome, Italy; 'Dermatology Unit, Fondazione Policlinico Universitario A. Gemelli-IRCCS, Rome, Italy
}

\section{Keywords}

Folliculitis decalvans - Cicatricial alopecia · Reflectance confocal microscopy · Noninvasive diagnosis

\begin{abstract}
Although trichoscopic criteria indicative of disease severity and inflammatory activity have been recently proposed, the potential use of reflectance confocal microscopy (RCM) in the evaluation of the inflammatory stage of FD has never been published to date. Our study investigated RCM features of 14 patients with a histopathological diagnosis of FD, evaluating clinical and trichoscopic findings. RCM findings were divided into 2 main patterns: "follicular" and "interfollicular." Our results suggest a potential role for RCM as a noninvasive, fast technique for a complementary investigation in the diagnostic process, as well as in the therapeutic management decision.

(c) 2020 S. Karger AG, Basel
\end{abstract}

Sara Mazzilli and Laura Vollono contributed equally to this work.

karger@karger.com

(C) 2020 S. Karger AG, Basel

www.karger.com/sad

Karger $\stackrel{2}{=}$

\section{Introduction}

Folliculitis decalvans (FD) is a rare form of scarring alopecia with a challenging clinical management. The pathogenesis is still not clearly elucidated; however, an abnormal immune activation triggered by a peculiar bacterial biofilm seems to play a key role $[1,2]$. Clinically, FD is characterized by crops of follicular pustules, hemorrhagic crusts, and tufted hairs (polytrichia), mainly in the vertex and occipital area, evolving to cicatricial alopecic patches on the scalp over time, with a typically chronic relapsing course [3]. Since hair loss alters patients' appearance, this disease impairs their psychoemotional wellness and quality of life, even in mild to moderate clinical forms [4].

Typical trichoscopic findings are tufted hairs surrounded by perifollicular hyperplasia arranged in a starburst pattern, and large follicular pustules with an emerging hair shaft. Pinpoint-like vessels and yellow tubular scaling have been also frequently observed, though not specific of FD [5, 6]. Diagnosis is guided by clinical and trichoscopic features and is based on histopathological examination. Histopathologically, early stages are characterized by a dense perifollicular neutrophilic infiltrate, 
Table 1. Trichoscopic FD findings $(n=14)$

\begin{tabular}{lc}
\hline Trichoscopic feature & Patients, $n(\%)$ \\
\hline Red patterns & \\
$\quad$ Perifollicular erythema & $13 / 14(92.8)$ \\
$\quad$ Perifollicular hemorrhages & $12 / 14(85.7)$ \\
Yellow structures & \\
$\quad$ Follicular pustules & $5 / 14(35.7)$ \\
$\quad$ Yellow tubular scaling & $11 / 14(78.5)$ \\
Yellow crusts & $10 / 14(71.4)$ \\
\hline
\end{tabular}

whereas a mixed intrafollicular and perifollicular infiltrate composed of neutrophils, lymphocytes, histiocytes, and plasma cells characterizes late stages, together with fused, multiple follicular units ("compound follicles" or multifollicular packs," responsible for the clinically evident follicular tufting) [7, 8].

Trichoscopic criteria predictive of disease severity and inflammatory activity have been recently proposed, suggesting a role for this noninvasive practice in the clinical management of the disease [9]. Reflectance confocal microscopy (RCM) is an in vivo noninvasive technique useful not only for the detection of skin tumors but also for identifying morphological and vascular changes in several inflammatory dermatoses $[10,11]$.

No data regarding the potential use of RCM in the evaluation of the inflammatory stage of FD have been published to date. Our study aimed to investigate RCM features of FD, suggesting this technique's potential role as an ancillary tool in the diagnosis and disease management.

\section{Materials and Methods}

This cross-sectional, multicenter study included consecutive patients with a confirmed histologic diagnosis of FD who attended the Institute of Dermatology, Catholic University-Fondazione Policlinico Universitario A. Gemelli IRCCS of Rome, Italy, and Dermatologic Clinic, University of Rome Tor Vergata, between June 1, 2019, and January 1, 2020. For each patient, clinical and dermatoscopic images of active lesions were acquired and recorded with the use of digital dermatoscopic devices (Dermaview DUAL, Tre T Medical snc, Cicciano, Italy). Trichoscopic features were evaluated according to the "trichoscopy activity scale" delineated by Saceda-Corralo et al. [9] in order to assess the inflammatory activity of the disease. RCM images were obtained with VivaScope ${ }^{\circledR} 1500$ (Caliber, New York, USA, distributed in Europe by MAVIG GmbH, Munich, Germany). Three VivaBlock ${ }^{\circledR}$ images (horizontal plane scan, $5 \times 5 \mathrm{~mm}$ ) at the level of the epidermis, DEJ and upper dermis, with several VivaStack ${ }^{\circledR}$ (RCM images taken at incremental depths from epidermis to dermis) in areas of particu-

The Usefulness of RCM in Diagnosis of Several Degrees of FD
Table 2. RCM FD adopted criteria

\begin{tabular}{lc}
\hline RCM image criterion & Patients, $n(\%)$ \\
\hline Follicular patterns & \\
$\quad$ Tufting & $9 / 14(64.2)$ \\
$\quad$ Perifollicular inflammatory infiltrates & $10 / 14(71.4)$ \\
Pustules & $8 / 14(57.1)$ \\
Interfollicular patterns & \\
$\quad$ Epidermal hyper/parakeratosis & $7 / 14(50)$ \\
Inflammatory cells & $14 / 14(100)$ \\
\hline
\end{tabular}

lar interest, were obtained. We proposed RCM criteria identifying "follicular patterns" (hair tufting, perifollicular inflammatory infiltrates, and pustules) and "interfollicular patterns" (epidermal hyper/parakeratosis and inflammatory infiltrates). The study was performed in conformity with the ethical guidelines of the Declaration of Helsinki.

\section{Results}

Fourteen patients ( 9 males and 5 females) affected by FD with a median age of 37 years (range 22-45 years) were enrolled. Dermoscopic evaluation was performed following the "trichoscopy activity scale" features described by Saceda-Corralo et al. [9]. The presence of "red patterns," namely, perifollicular erythema, and perifollicular hemorrhages was disclosed in most of the lesions evaluated, while "yellow structures" such as yellow tubular scaling and yellow crusts were observed at a less percentage. Pustules were identified in one-third of cases (Table 1). RCM criteria related to the inflammatory activity of FD were divided into 2 groups. The first group regarding "follicular patterns" included findings like tufting, pustules, and perifollicular inflammatory infiltrates. Tufting, defined as the merging of multiple hair ( $>5$ hair shafts) from the same follicular unit, was observed in $9 / 14$ cases. Pustules and perifollicular inflammatory infiltrates were observed, respectively, in 8/14 and 10/14 cases. At RCM examination, pustules appeared as round to oval structures constituted by reflective amorphous material, correlated with epidermal and superficial dermal perifollicular inflammatory infiltrate. The second group named "interfollicular patterns" included epidermal hyper/parakeratosis and inflammatory cells (Table 2). The RCM correlate of hyperkeratosis is represented by a thickened epidermis, while that of parakeratosis is associated to highly refractile, polygonal structures at the level of the stratum corneum, similar to or smaller in size than normal kera- 

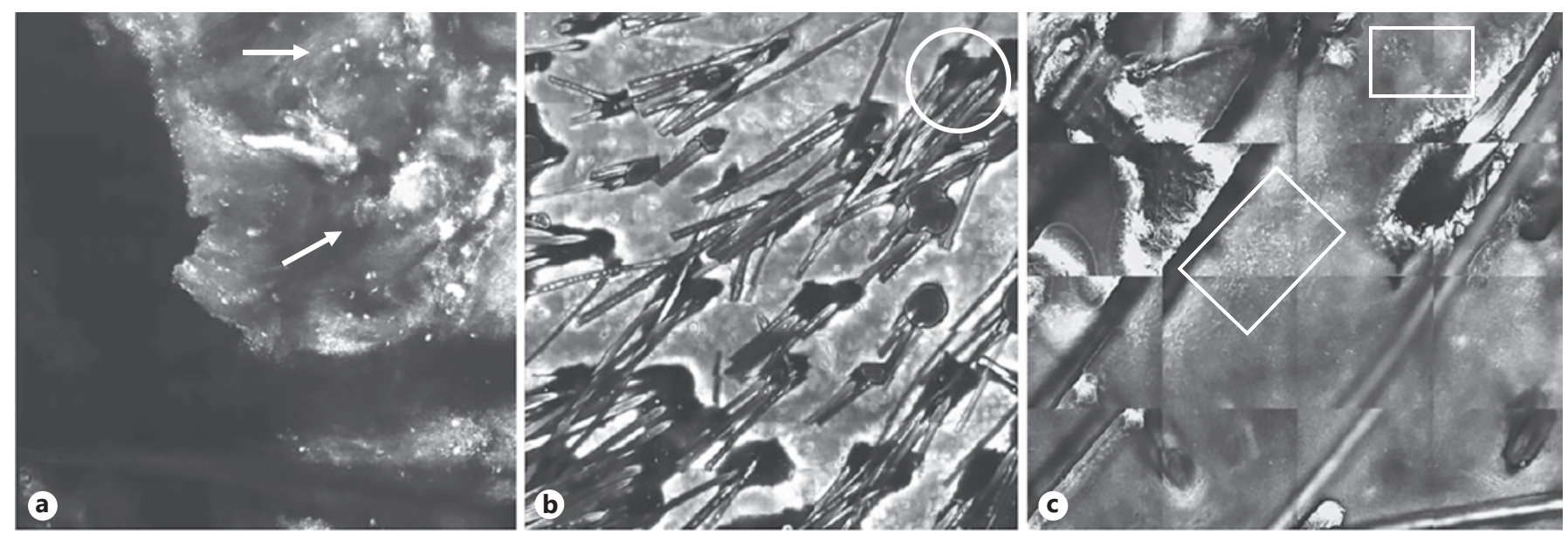

Fig. 1. Mosaics of epidermis (VivaScope ${ }^{\circledR} 1500$ ) highlighting confocal findings of active lesions in FD. a Single and aggregated inflammatory cells (arrow). b Tufting involving numerous follicular units with a perifollicular hyper/parakeratosis (circle), associated with peri- and interfollicular inflammatory infiltrates (c).
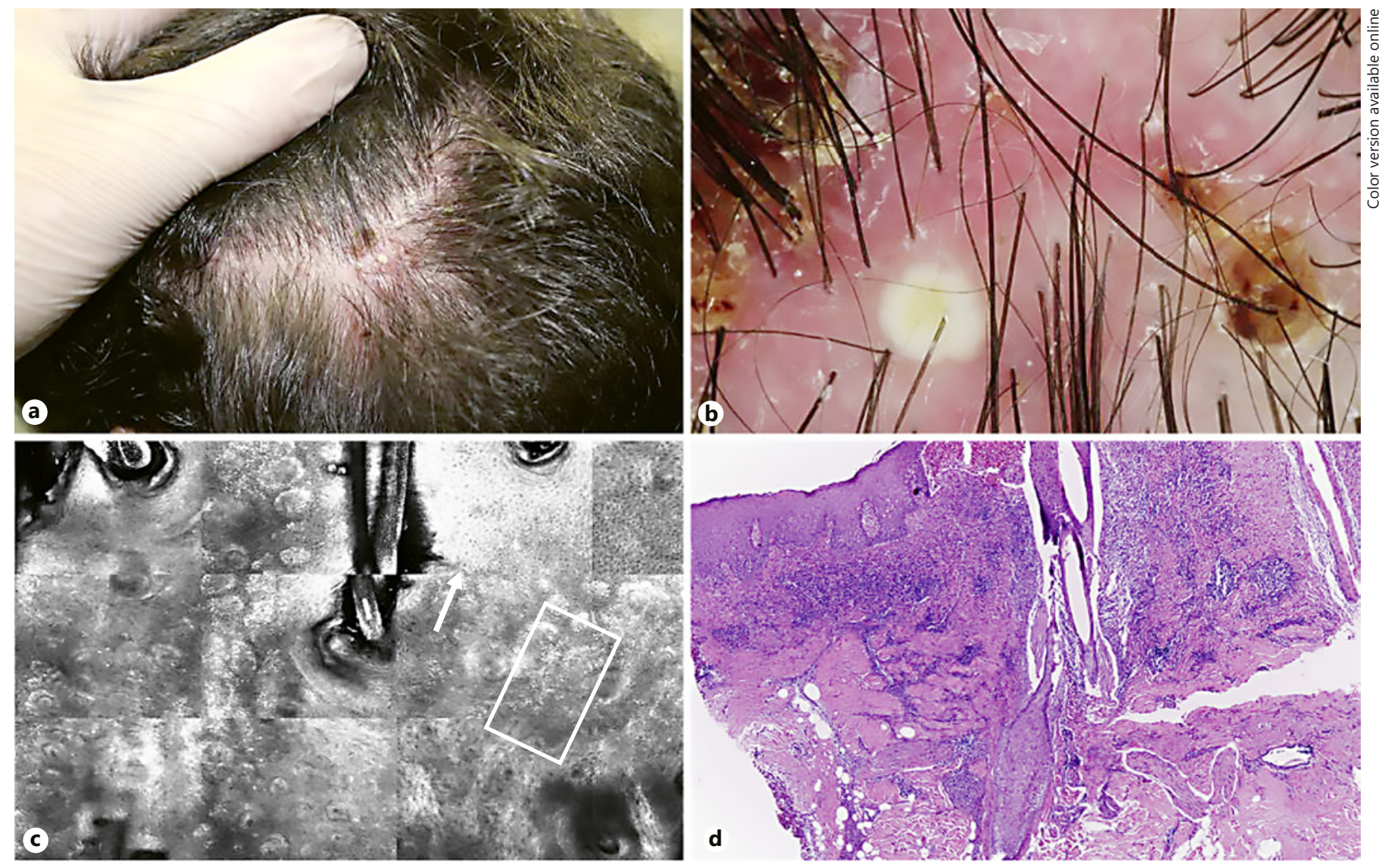

Fig. 2. Clinical, dermatoscopic, confocal and histopathological features of FD. a Clinical image of an active lesion localized on temporo-parietal area, showing dermatoscopy follicular pustules, yellow tubular scaling and perifollicular haemorrhages (b). c RCM identifies inflammatory cells (with an inter- and perifollicular distribution (square) associated with hyper/parakeratosis (arrow). d H\&E staining confirms the presence of a diffuse inflammatory infiltrate. 
tinocytes (corresponding to the remains of the keratinocyte nuclei). Inflammatory cells were evaluated like single or aggregated round-to-polygonal spots $(<10 \mu \mathrm{m})$, showing mid-to-high refractivity, visible at the level of the stratum spinosum and located in the interkeratinocytic spaces (Fig. 1a-c). Hyper/parakeratosis and inflammatory cells were observed in $7 / 14$ patients and 14/14 patients, respectively (Fig. 2).

\section{Discussion}

FD, first described by Quinquaud in 1888 , is a primary cicatricial neutrophilic alopecia. Topical and oral antibiotics are the mainstay of treatment; however, response is variable and often unsatisfactory. In a multicenter study including 60 patients, Miguel-Gómez et al. [12] proposed a tailored therapy based on patients' clinical conditions. Incisional biopsy is required to make a diagnosis; however, evaluation of the disease severity through consecutive biopsies is often rejected by patients and bear significant practical and economic implications, and thus is not recommended. Hence, identification of noninvasive methods capable of assessing the activity of FD and guiding clinicians in the therapeutic management may be useful. Ardigò et al. [13] investigated RCM features of several hair diseases; however, no data regarding the use of RCM in FD have been published to date. We add to the literature our experience of 14 cases of histologically confirmed FD evaluated with RCM. In our sample, epidemiologic data are in line with evidence of the literature, demonstrating male predominance and the mean young age of FD-affected patients [3]. At RCM examination, we assessed the simultaneous presence of highly reflective tubular structures varying in length and rising up from the same follicular unit, representing the related feature of tricoscopic clue of tufting. Round to oval bright structures surrounded with a regular rim represent the RCM counterpart of the pustules observed at trichoscopy. Among RCM interfollicular patterns, the finding of hyper/parakeratosis likely corresponds to scales and crusting detected at trichoscopy.

The role of trichoscopy (dermoscopy of hair and scalp) as an auxiliary tool in the diagnosis of hair diseases is widely recognized. Recently, Saceda-Corralo et al. [9] associated trichoscopic findings of FD with inflammatory activity of the disease, investigating its early stage and giving complementary insights to the trichoscopic features previously described by Fernández-Crehuet et al. [6] regarding the extension of cicatricial patches of alopecia in the advanced course of FD $[5,6]$. Although we considered "trichoscopic activity scale" in the evaluation of our lesions, it was difficult to create a perfect match between trichoscopic and confocal criteria, since RCM offers an in vivo image with a cellular detailed resolution more similar to histology, pointing out some interesting considerations. First, it could suggest a potential role for RCM as a noninvasive, fast technique for a complementary investigation in the diagnostic process. Furthermore, being able to evaluate the extension of inflammatory infiltrate, not only with a perifollicular localization but also as single infla006Dmatory cells with interfollicular distribution, it might allow the detection of flare-ups, improvements, or worsening at a preclinical stage, supporting clinicians in the therapeutic management. Our sample size did not allow us to create a proper grading system for disease severity, but the creation of a detailed "confocal activity scale" could be useful. Moreover, RCM could be managed as a supportive technique in addition to trichoscopy, for the identification of the optimal site for scalp biopsy, suggesting a "combined dermoscopy-RCM-guided biopsy" approach [14]. These suggestions, however, remain speculative and require further investigation to assess the potential benefits of RCM to those aims.

Limitations of our observations are the small sample size and the impossibility to characterize the cytomorphological features of the inflammatory infiltrate due to limited RCM in vivo resolution. It should be also mentioned that RCM is an expensive technique, time-consuming and not available everywhere.

\section{Statement of Ethics}

Approval from the Institutional Review Board was obtained prior to initiation of the study. All procedures performed in studies involving human participants were in accordance with the 1964 Helsinki Declaration and its later amendments or comparable ethical standards. Informed consent was obtained from all individual participants included in the study.

\section{Disclosure Statement}

The authors have no conflicts of interest to declare.

\section{Funding Sources}

No funding was received for this study. 


\section{Author Contributions}

M.S, V.L., F.T., S.C., K.P., and L.B. have contributed to the conception and design of the work; the acquisition, analysis, and interpretation of the data; and draft and revision of the manuscript.
All the authors have revised and approved the final version of the manuscript and make themselves responsible for the accuracy and integrity of the work as a whole.

\section{References}

1 Eyraud A, Milpied B, Thiolat D, Darrigade AS, Boniface K, Taïeb A. et al. Inflammasome activation characterizes lesional skin of folliculitis decalvans. Acta Derm Venereol. 2018 Jun;98(6):570-5.

2 Matard B, Donay JL, Resche-Rigon M, Tristan A, Farhi D, Rousseau C. et al. Folliculitis decalvans is characterized by a persistent, abnormal subepidermal microbiota. Exp Dermatol. 2019 Mar;29(3):295-8.

3 Vañó-Galván S, Molina-Ruiz AM, Fernández-Crehuet P, Rodrigues-Barata AR, AriasSantiago S, Serrano-Falcón C. et al. Folliculitis decalvans: a multicentre review of 82 patients. J Eur Acad Dermatol Venereol. 2015 Sep; 29(9):1750-7.

4 Pindado-Ortega C, Saceda-Corralo D, MiguelGómez L, Buendía-Castaño D, FernándezGonzález P, Moreno-Arrones OM. et al. Impact of folliculitis decalvans on quality of life and subjective perception of disease. Skin Appendage Disord. 2018 Jan;4(1):34-6.

5 Rakowska A, Slowinska M, Kowalska-Oledzka E, Warszawik O, Czuwara J, Olszewska M. et al. Trichoscopy of cicatricial alopecia. J Drugs Dermatol. 2012 Jun;11(6):753-8.

6 Fernández-Crehuet P, Vañó-Galván S, Molina-Ruiz AM, Rodrigues-Barata AR, SerranoFalcón C, Martorell-Calatayud A. et al. Trichoscopic features of folliculitis decalvans: results in 58 patients. Int J Trichology. 2017 Jul-Sep;9(3):140-1.

7 Stefanato CM. Histopathology of alopecia: a clinicopathological approach to diagnosis. Histopathology. 2010 Jan;56(1):24-38.

8 Egger A, Stojadinovic O, Miteva M. Folliculitis decalvans and lichen planopilaris phenotypic spectrum: a series of 7 new cases with focus on histopathology. Am J Dermatopathol. 2020 Mar;42(3):173-7.

9 Saceda-Corralo D, Moreno-Arrones OM, Rodrigues-Barata R, Rubio-Lombraña M, MirBonafé JF, Morales-Raya C. et al. Trichoscopy activity scale for folliculitis decalvans. J Eur Acad Dermatol Venereol. 2020 Feb;34(2): e55-7.

10 Longo C, Zalaudek I, Argenziano G, Pellacani G New directions in dermatopathology: in vivo confocal microscopy in clinical practice. Dermatol Clin. 2012 Oct;30(4):799-814.

11 Ardigo M, Longo C, Gonzalez S. Multicentre study on inflammatory skin diseases from The International Confocal Working Group: specific confocal microscopy features and an algorithmic method of diagnosis. Br J Dermatol. 2016 Aug;175(2):364-74.

12 Miguel-Gómez L, Rodrigues-Barata AR, Molina-Ruiz A, Martorell-Calatayud A, Fernández-Crehuet P, Grimalt R. et al. Folliculitis decalvans: effectiveness of therapies and prognostic factors in a multicenter series of 60 patients with long-term follow-up. J Am Acad Dermatol. 2018 Nov;79(5):878-83.

13 Ardigò M, Agozzino M, Franceschini C, Donadio C, Abraham LS, Barbieri L. et al. Reflectance confocal microscopy for scarring and non-scarring alopecia real-time assessment. Arch Dermatol Res. 2016 Jul;308(5): 309-18.

14 Miteva M, Tosti A. Dermoscopy guided scalp biopsy in cicatricial alopecia. J Eur Acad Dermatol Venereol. 2013 Oct;27(10):1299-303. 